\title{
Mapping of the second locus for the Van der Woude syndrome to chromosome $1 \mathrm{p} 34$
}

\author{
Hannele Koillinen ${ }^{1,8,10}$, Fung Ki Wong ${ }^{2}$, Jorma Rautio ${ }^{3}$, Vesa Ollikainen ${ }^{4}$, Agneta Karsten ${ }^{5}$, \\ Ola Larson ${ }^{6}$, Bin Tean Teh ${ }^{7}$, Jan Huggare ${ }^{5}$, Päivi Lahermo ${ }^{4}$, Catharina Larsson ${ }^{2}$ and \\ Juha Kere*,4,9
}

\begin{abstract}
${ }^{1}$ Department of Medical Genetics, University of Helsinki, Finland; ${ }^{2}$ Department of Molecular Medicine, Karolinska Hospital, Stockholm, Sweden; ${ }^{3}$ Cleft Center, University Hospital of Helsinki, Finland; ${ }^{4}$ Finnish Genome Center, University of Helsinki, Finland; ${ }^{5}$ Department of Orthodontics, Institution of Odontology, Karolinska Institute, Stockholm, Sweden; ${ }^{6}$ Department of Reconstructive Plastic Surgery, Karolinska Hospital, Stockholm, Sweden; ${ }^{7}$ Van Andel Research Institute, Grand Rapids, Michigan, USA; ${ }^{8}$ Department of Medical Genetics, Väestöliitto, Family Federation, Helsinki, Finland; ${ }^{9}$ Department of Medical Genetics, University of Turku, Finland; ${ }^{10}$ Department of Pediatric Neurology, University of Turku, Finland
\end{abstract}

The Van der Woude syndrome (VWS) is a dominantly inherited developmental disorder characterized by pits and/or sinuses of the lower lip, cleft lip and/or cleft palate. It is the most common cleft syndrome. VWS has shown remarkable genetic homogeneity in all populations, and so far, all families reported have been linked to 1q32-q41. A large Finnish pedigree with VWS was recently found to be unlinked to 1q32-q41. In order to map the disease locus in this family, a genome wide linkage scan was performed. A maximum lod score of 3.18 was obtained with the marker D1S2797, thus assigning the disease locus to chromosomal region 1p34. By analyses of meiotic recombinants an $\sim 30 \mathrm{cM}$ region of shared haplotypes was identified. The results confirm the heterogeneity of the VWS syndrome, and they place the second disease locus in 1p34. This finding has a special interest because the phenotype in VWS closely resembles the phenotype in non-syndromic forms of cleft lip and palate. European Journal of Human Genetics (2001) 9, 747-752.

Keywords: Van der Woude syndrome; cleft lip and palate; cleft palate; lip pits; linkage analysis; chromosome 1

\section{Introduction}

The Van der Woude syndrome (VWS) (OMIM 119300) is a dominantly inherited developmental disorder that was first described by Anne Van der Woude in $1954 .^{1}$ The hallmarks of VWS are pits and/or sinuses of the lower lip, cleft lip and/or cleft palate $(\mathrm{CL} / \mathrm{P}, \mathrm{CP})$. VWS was the most common recognised syndrome among Finnish cleft patients. ${ }^{2}$ The penetrance has been estimated to be $\sim 90 \%$ with both sexes equally affected. ${ }^{3-5}$ Lip pits are present in $\sim 80 \%$, clefts in $\sim 50 \%$ and hypodontia in $25 \%$ of the gene

\footnotetext{
*Correspondence: Dr Juha Kere, Finnish Genome Center, PO BOX 63 (Haartmanink. 8), 00014 University of Helsinki, Finland.

Tel: +358 9 19126538; fax: +358 9 19126789; E-mail: juha.kere@helsinki.fi Received 2 April 2001; revised 12 July 2001; accepted 31 July 2001
}

carriers. ${ }^{1,6-9}$ The incidence of VWS is estimated at $1 / 34000$ livebirths, ${ }^{6}$ and the mutation rate at $\sim 1.8 \times 10^{-5}$. $^{3}$ A recent study suggested the possibility of a common origin for a mutation responsible for the majority of VWS cases. ${ }^{10}$

In 1987 Bocian et al. reported a patient with lip pits and a deletion in 1q32-q41, and subsequently Murray et al. found linkage between VWS and markers from the same region. ${ }^{4,11}$ Following extended linkage analyses the VWS region was narrowed down to $4.1 \mathrm{cM}$ in 1995 and further to $1.6 \mathrm{~cm}$ in 1996. ${ }^{12,13}$ In that region there are at least 15 confirmed genes, 9 putative genes and 3 pseudogenes. ${ }^{14}$ Microdeletions in 1q32-q41 have also been reported in families with VWS. ${ }^{15,16}$ A possible modifying locus at 17p11.2-p11.1 was reported when a large Brazilian family was analysed. ${ }^{17}$ An allele in this locus would enhance the probability of CP in an individual 
carrying also a defect in the VWS locus. The popliteal pterygium syndrome (OMIM 119500) was recently found to be linked to the VWS, suggesting that this syndrome might well be allelic to VWS region. ${ }^{18,19}$

In the Finnish population VWS is found in $\sim 2 \%$ of all patients with cleft. ${ }^{6}$ Evidence of genetic heterogeneity was first noted among Finnish VWS families by Wong et al. who analysed five pedigrees and found that two of them were unlinked to $1 \mathrm{q} 32-\mathrm{q} 41 .^{20}$ These findings motivated a genome-wide scan to look for evidence for a second disease locus.

I
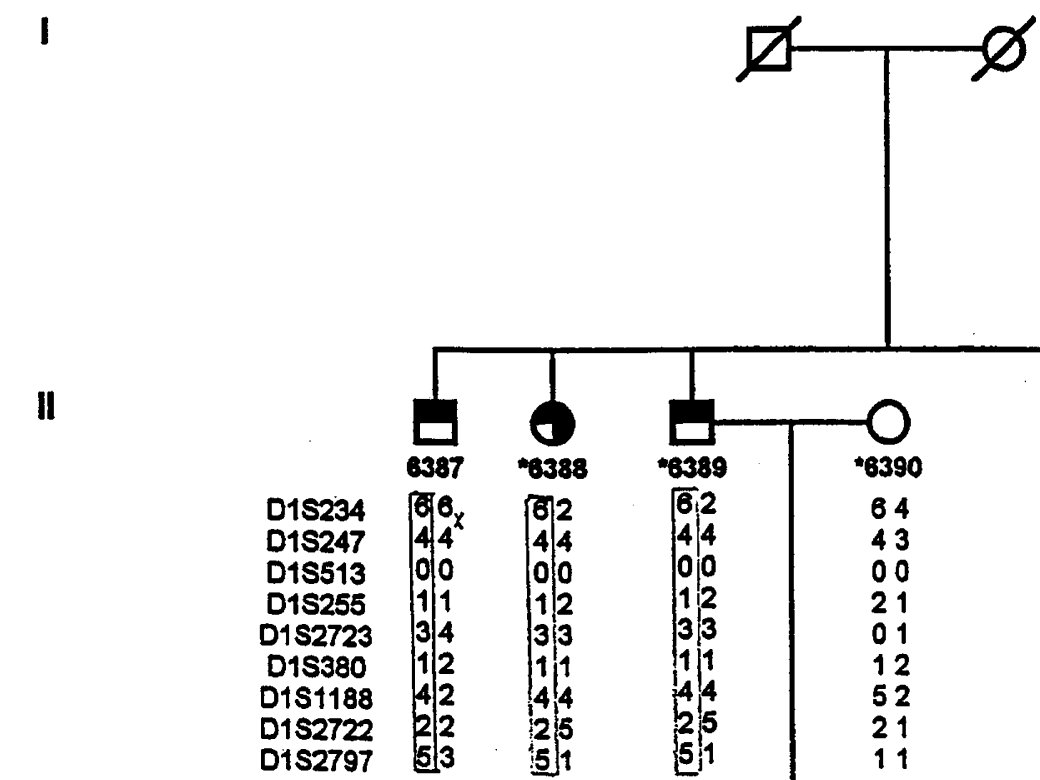

III

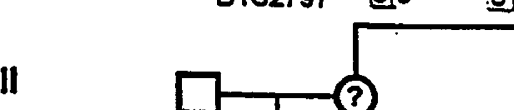

IV

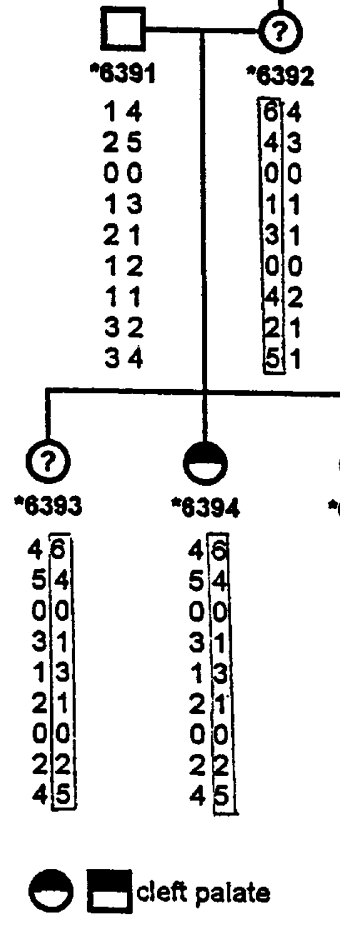

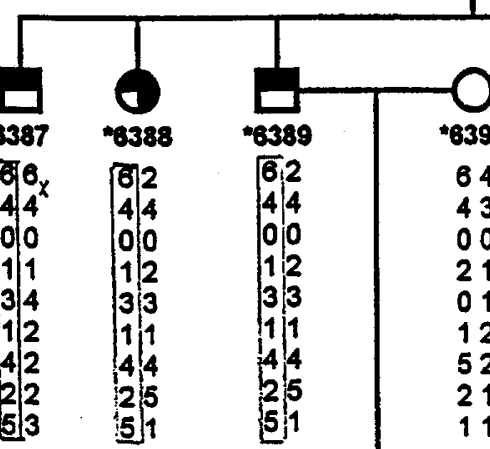

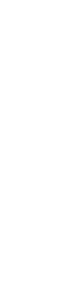

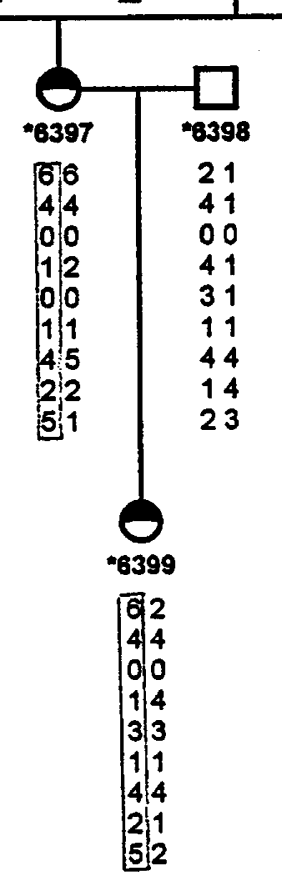

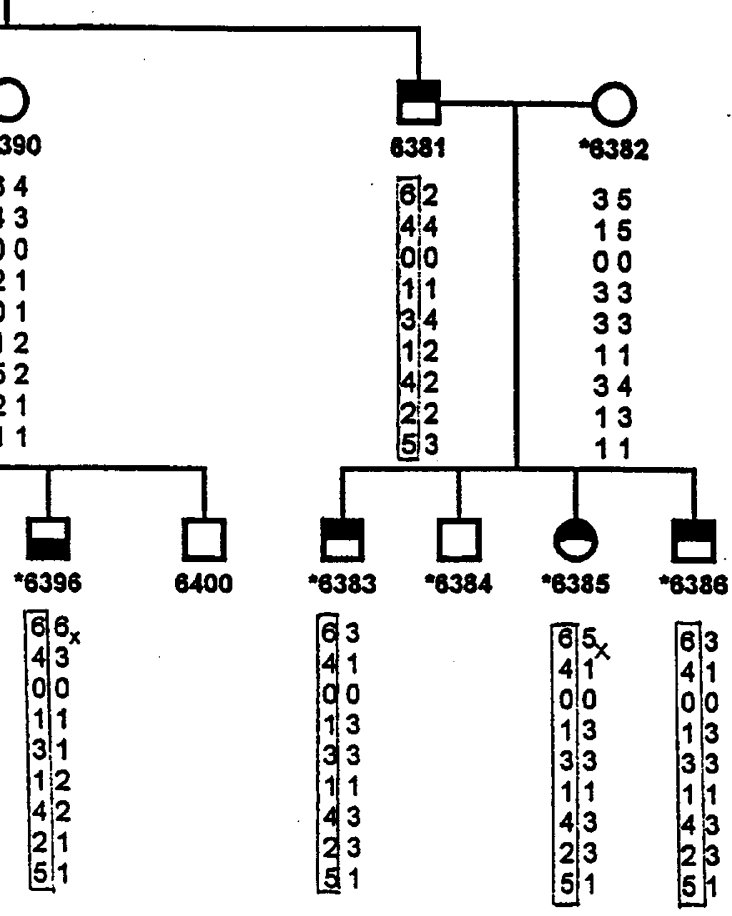

Figure 1 Pedigree of the VWS family and haplotypes of nine linked markers in 1p34. Marker D1S2797 gave the highest LOD score of 3.18. The disease associated haplotype is marked with a box, and meiotic recombination events detected are indicated by an ' $x$ '. The allele ' 0 ' indicates that no clear genotype could be assured. No samples were available from individuals 6384 and 6400 . Individuals marked with an asterisk were carefully re-examined in purpose of confirming the diagnosis. 


\section{Patients and methods}

Family 0057 consists of nine unaffected and 11 affected individuals with a diagnosis of VWS (Figure 1). The pedigree is consistent with autosomal dominant inheritance. The individuals that were re-examined by JR in this study are marked with an asterisk. Originally, the VWS diagnosis was established by a dysmorphologist based on the dominant inheritance pattern and clinical examination of individual 6396 who has lip pits but no associated cleft (Figure 2). Careful clinical re-examination of the family members also supported the absence of additional syndromic features that would suggest a diagnosis other than VWS. All the members of the family have managed well in their employment and social life.

Family 0062 consists of an affected father and two affected children. The father has cleft lip and palate with lip pits but both children have cleft palate only and no pits.

DNA was extracted from peripheral leukocytes using standard methods. In family 0057 the genome scan was performed at the Finnish Genome Center with 381 polymorphic microsatellite markers from ABI PRISM Linkage Mapping Set-MD10 (Applied Biosystems). For the second stage of analysis, six additional markers (D1S247, D1S513, D1S2723, D1S380, D1S1188, D1S2722) within the region flanked by markers D1S234 and D1S2797 were genotyped. The positions of these markers were ascertained from the Marshfield comprehensive human genetic map (http:// www.marshmed.org/genetics/).

In family 0062, the chromosome 1 was analysed with 26 polymorphic markers. In our previous study, seven markers

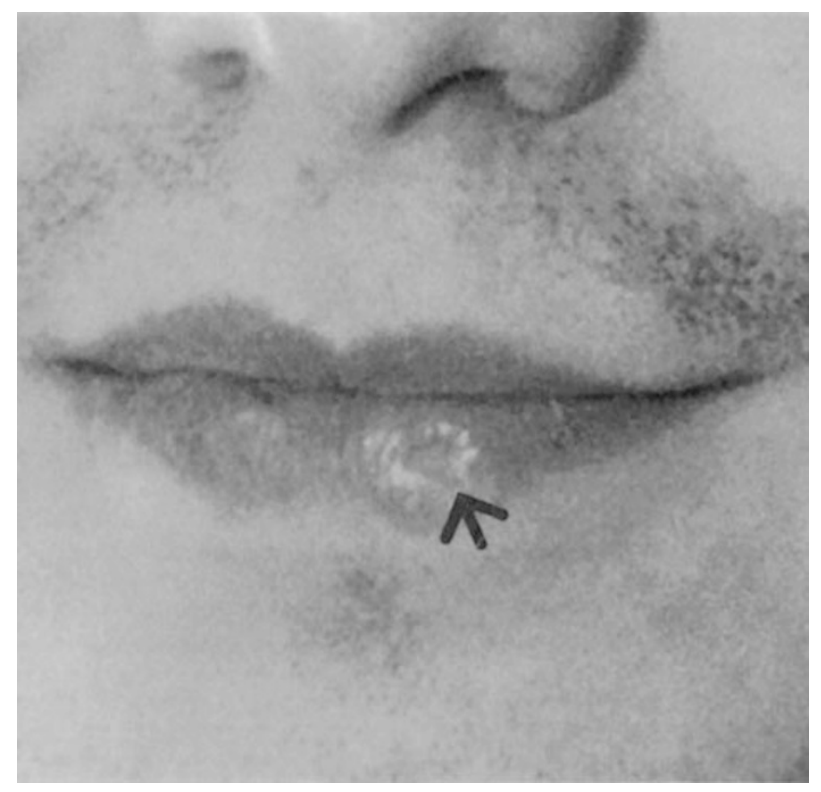

Figure 2 Illustration of lip pits in individual 6396 who does not have any kind of associated cleft. in the critical VWS region with length of $2.5 \mathrm{cM}$ were analysed. $^{20}$

Multipoint linkage analyses were performed with the Genehunter program. ${ }^{21}$ The disease allele frequency was estimated to be $2 \times 10^{-5}{ }^{6}$ The inheritance model was dominant with $95 \%$ penetrance, and the phenocopy frequency was set at $1 \times 10^{-6}$.

\section{Results}

In family 0057, a $30 \mathrm{cM}$ region in chromosome 1p34 showed the highest LOD scores in the initial scan. The genotyping of additional markers in this region increased the maximum LOD score to 3.18 at marker D1S2797 with theta $=0$ and information content of 0.91 (Table 1, Figure 3). This score is close to the theoretical maximum in this pedigree achieved by simulation using SLINK with 200 replicates (data not shown). ${ }^{22}$ Changing the phenocopy rate to $1 \times 10^{-3}$ did not have any affect to LOD scores. Lowering the penetrance to $90 \%$ gave the maximum LOD score 3.16. The LOD scores in all other regions of the genome fell below 1.49 .

In family 0062 the affected father was homozygous for all the markers from the critical $2.5 \mathrm{cM}$ VWS region. Both the children also share the particular haplotype but the analysis of all other 26 markers covering the whole chromosome 1 excluded the linkage (Figure 4). Markers from 1 p34 region were not tested because the initial analysis did not find any suggestive linkage in 1p34 (Figure 4).

Table 1 LOD scores for linkage of the VWS syndrome to the 1 p34 region in family 0057.

\begin{tabular}{lccc}
\hline Position & LOD score & p-value & Information \\
\hline 39.39 & 1.853 & 0.006393 & 0.770 \\
50.68 & 2.696 & 0.000763 & 0.770 \\
58.06 & 2.993 & 0.000488 & 0.796 \\
58.51 & 3.003 & 0.000488 & 0.792 \\
59.41 & 3.023 & 0.000488 & 0.788 \\
59.86 & 3.034 & 0.000488 & 0.788 \\
62.13 & 3.087 & 0.000488 & 0.803 \\
63.27 & 3.115 & 0.000488 & 0.822 \\
64.41 & 3.143 & 0.000488 & 0.852 \\
65.91 & 3.172 & 0.000488 & 0.901 \\
66.65 & 3.174 & 0.000488 & 0.900 \\
67.38 & 3.176 & 0.000488 & 0.902 \\
68.70 & 3.177 & 0.000488 & 0.900 \\
70.02 & 3.180 & 0.000488 & 0.904 \\
71.13 & 3.181 & 0.000488 & 0.907 \\
72.03 & 3.179 & 0.000488 & 0.908 \\
73.57 & 3.176 & 0.000488 & 0.905 \\
74.85 & 3.173 & 0.000488 & 0.898 \\
76.13 & 3.171 & 0.000488 & 0.896 \\
78.66 & 3.139 & 0.000488 & 0.862 \\
81.20 & 3.113 & 0.000488 & 0.845 \\
83.74 & 3.094 & 0.000488 & 0.842 \\
86.28 & 3.083 & 0.000488 & 0.851 \\
88.81 & 3.078 & 0.000488 & 0.879 \\
\hline & & &
\end{tabular}




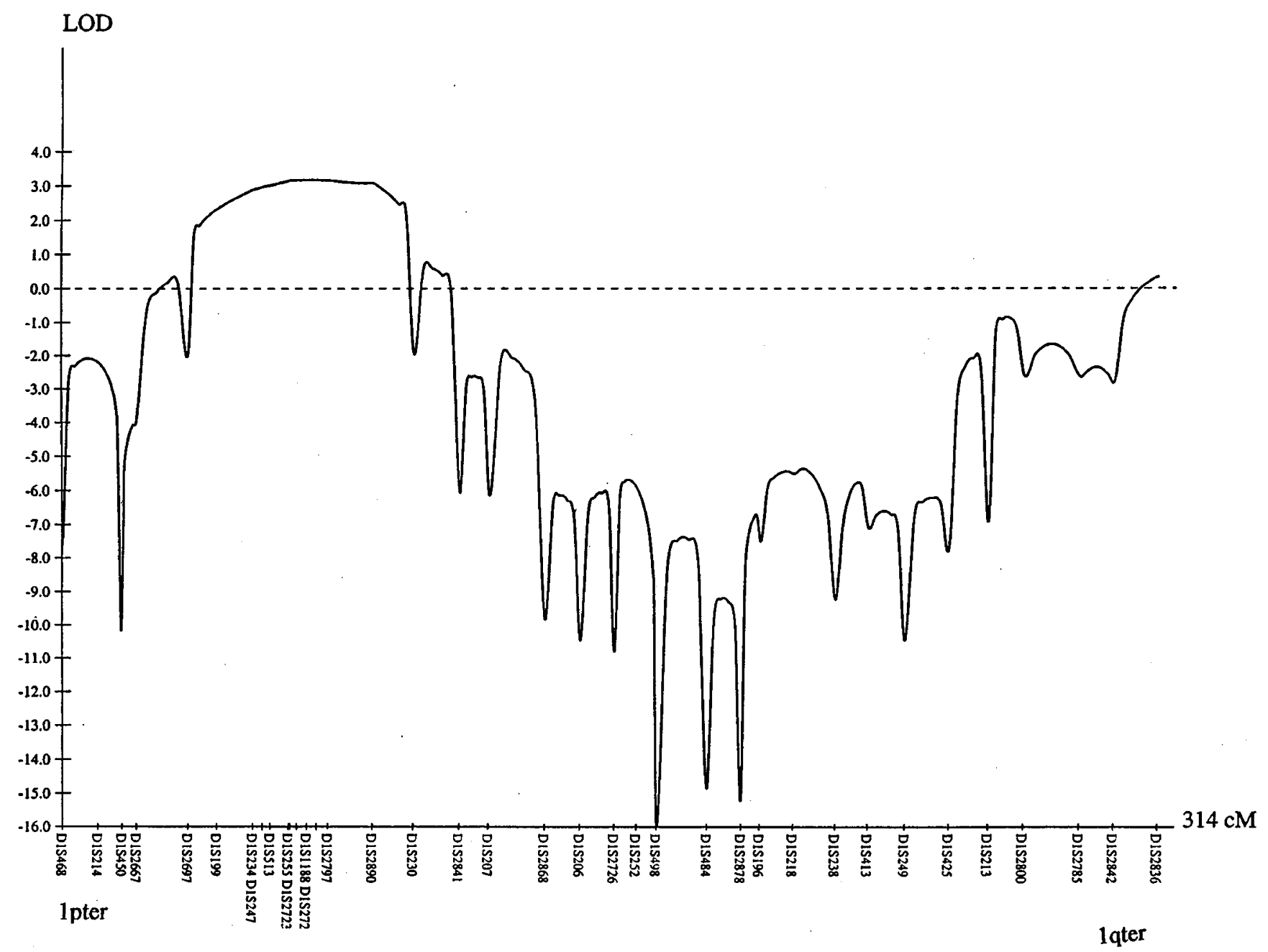

Figure 3 Maximum LOD score values obtained from the whole chromosome 1 using 37 polymorphic markers in family 0057.

\section{Discussion}

VWS has shown remarkable genetic homogeneity in all populations. $^{4,13,17,19,23}$ Our surprising finding of a large family that was unlinked to 1q32-q41 prompted a search for a possible second locus, that was mapped in this study to chromosomal region $1 \mathrm{p} 34$. The clinical features of family 0057 are typical for VWS with one exception: usually $\mathrm{CL} / \mathrm{P}$ is twice as common as cleft palate, but in this family nine individuals have $\mathrm{CP}$ and only one has $\mathrm{CL} / \mathrm{P}$. One explanation to this can be that Finland is one of the few countries in the world where the incidence of $\mathrm{CP}$ is higher than the incidence of $\mathrm{CL} / \mathrm{P}^{24}$ This may suggest that other modifying loci or possibly extrinsic factors are involved in determining the exact phenotype. It is also worth noticing that in this family one individual only has lip pits, when usually lip pits are present in $80 \%$ of VWS patients. This observation together with the cleft palate phenotype may imply a new subtype or variation of the Van der Woude syndrome.
Our statistical analysis did not find linkage to 1q32-q41 nor 1 p34 in family 0062. Instead, one explanation is that the affected father is a true homozygous for VWS causing mutation in 1q32-q41. This suggestion is supported by the fact that the part of the haplotype (3-5-3-5-3) is identical to the haplotypes of two other previously reported Finnish VWS families. $^{20}$

The members of family 0057 shared a $30 \mathrm{cM}$ haplotype with no crossing-overs to allow more precise mapping. Narrowing of the critical region will thus require identification of additional rare families in which the syndrome is linked to $1 \mathrm{p} 34$. However, for those VWS families in which only 1q32-q41 was analysed, it might be worthwhile to reassess the new region in 1 p34.

Several interesting candidate genes map to the linked region in 1p34. CRTM (cartilage matrix protein, matrilin-1) has already been excluded as a major gene in non-syndromic $\mathrm{CL} / \mathrm{P}$ families. ${ }^{25}$ Cranial neural crest defects have been 


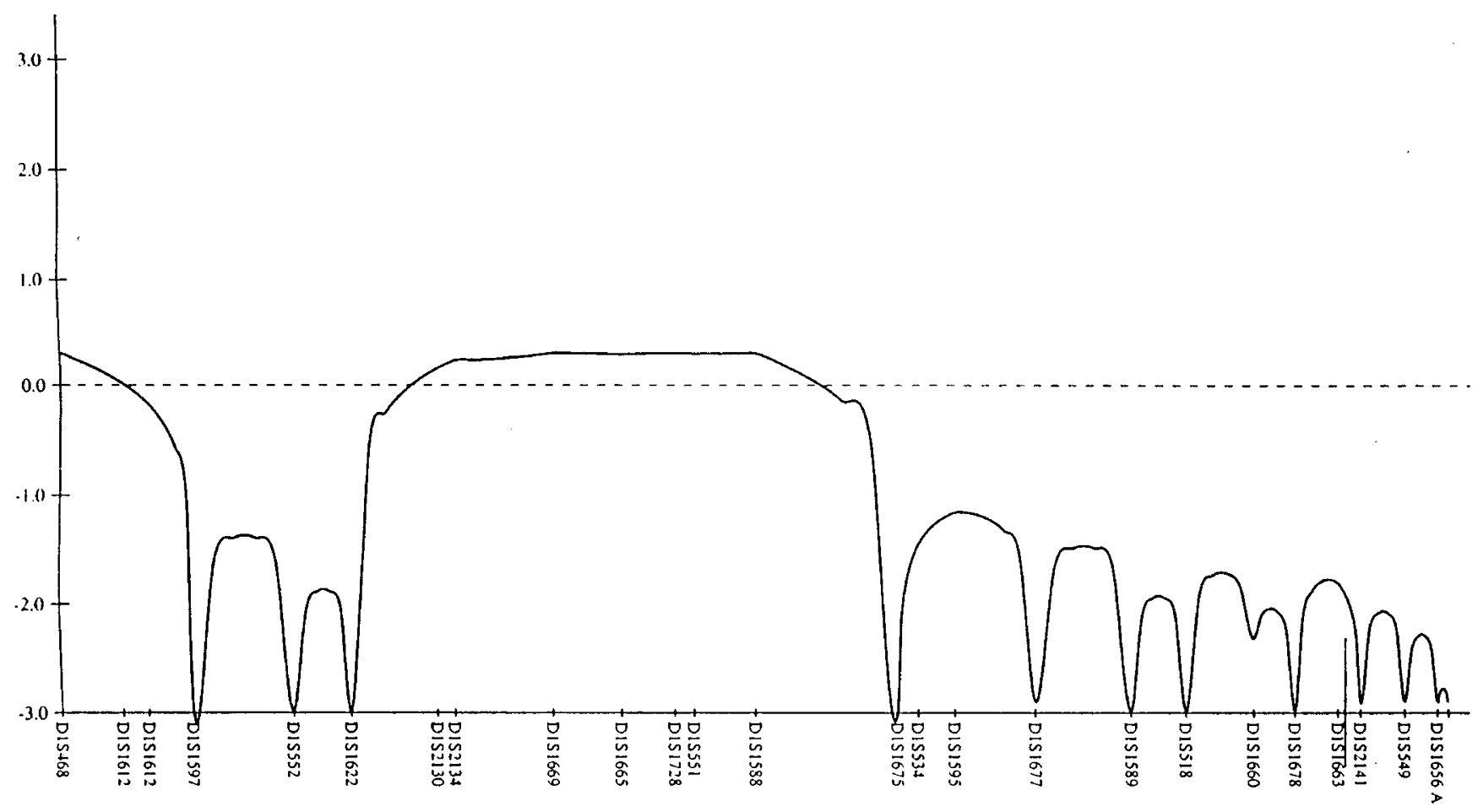

Figure 4 Maximum LOD score values from the whole chromosome 1 in family 0062.

reported in endothelin-A receptor-deficient mice, ${ }^{26}$ and endothelin 2 maps to the region we implicate in family 0057. Mutations in COL9A2 cause multiple epiphyseal dysplasia, ${ }^{27}$ and cleft palate is seen in many skeletal dysplasias. Sequencing of this part of the chromosome is in progress and will reveal new additional candidate genes for mutation screening.

Van der Woude syndrome is of special interest because the phenotype so closely resembles non-syndromic forms of both cleft lip and palate. Non-syndromic cleft palate has a high incidence in Finland; about one 1.01 per thousand livebirths. ${ }^{2}$ So far, no convincing linkage has been established, although a higher recurrence risk for $1^{\circ}$ relatives have been observed. ${ }^{28,29}$ The identification of a second VWS locus in 1p34 thus provides a new candidate location for nonsyndromic cleft palate which should be further assessed in such pedigrees.

\section{Acknowledgements}

We thank all members of the affected kindred for their participation. This study was supported by the Sigrid Juselius Foundation, the Academy of Finland, the Helsinki University Hospital research funds and the Swedish Medical Research Council.

\section{References}

1 Van der Woude A: Fistula labii inferioris congenita and its association with cleft lip and palate. Am J Hum Genet 1954; 6: $244-256$.
2 Lilius P: Epidemiology of cleft lip and palate in Finland $1975-$ 1985. PhD thesis, Cleft Center, University Central Hospital of Helsinki, Helsinki, Yliopistopaino, 1992.

3 Burdick $\mathrm{AB}$, Bixler D, Puckett C: Genetic analysis in families with Van der Woude syndrome. J Craniofac Genet Dev Biol 1985; 5: $181-208$.

4 Murray JC, Nishimura DY, Buetow KH et al: Linkage of an autosomal dominant clefting syndrome (Van der Woude) to loci on chromosome 1q. Am J Hum Genet 1990; 46: 486-491.

5 Onofre MA, Brosco HB, Taga R: Relationship between lower-lip fistulae and cleft lip and/or palate in Van der Woude syndrome. Cleft Plate Craniofac J 1997; 34: 261 - 265.

6 Rintala AE, Ranta R: Lower lip sinuses. epidemiology of microforms and transverse sulci. Br J Plast Surg 1981; 34: 26-30.

7 Burdick AB: Genetic epidemiology and control of genetic expression in Van der Woude syndrome. J Craniofac Genet Dev Biol 1986; (Suppl) 2: 99-105.

8 Schinzel A, Kläusler $\mathrm{M}$ : The Van der Woude syndrome (dominantly inherited lip pits and clefts). J Med Genet 1986; 23: $291-294$

9 Kläusler M, Schinzel A, Gnoinski W, Hotz M, Perko M: Dominant vererbte Unterlippenfisteln und Gesichtsspalten (Van-der-Woude-Syndrom). Schweiz Med Wschr 1987; 117: $127-134$.

10 Beiraghi S, Miller-Chisholm A, Kimberling WJ et al: Confirmation of linkage of Van der Woude syndrome to chromosome 1q32: evidence of association with STR alleles suggests possible unique origin of the disease mutation. J Craniofac Genet Dev Biol 1999; 19: $128-34$.

11 Bocian M, Walker AP: Lip pits and deletion 1q32-41. Am J Med Genet 1987; 26: $437-443$.

12 Sander A, Murray JC, Scherpbier-Heddema Tet al: Microsatellitebased fine mapping of the van der Woude syndrome locus to an interval of 4.1 cM between D1S245 and D1S414. Am J Hum Genet 1995; 56: 310-318. 
13 Schutte BC, Sander A, Malik M, Murray JC: Refinement of the Van der Woude gene location and construction of a 3.5-Mb YAC contig and STS maps spanning the critical region in 1q32-41. Genomics 1996; 36: 507-514.

14 Schutte BC, Bjork BC, Coppage KB et al: A preliminary gene map for the Van der Woude syndrome critical region derived from $900 \mathrm{~kb}$ of genomic sequence at 1q32-q41. Gen Res 2000; 10: 81 94.

15 Sander A, Schmelzle R, Murray J: Evidence for microdeletion in 1q32-41 involving the gene responsible for Van der Woude syndrome. Hum Mol Genet 1994; 3: 575-578.

16 Schutte BC, Basart AM, Watanabe Y et al: Microdeletions at chromosome bands 1q32-q41 as a cause of Van der Woude syndrome. Am J Med Genet 1999; 84: 145 - 150.

17 Sertie AL, Sousa AV, Stenman S, Pavanello RC, Passos-Bueno MR: Linkage analysis in a large Brazilian family with van der Woude syndrome suggests the existence of a susceptibility locus for cleft palate at 17p11.2-11-1. Am J Hum Genet 1999; 65: 433-440.

18 Lees MM, Winter RM, Malcolm S, Saal HM, Chitty L: Popliteal pterygium syndrome: a clinical study of three families and report of linkage to the Van der Woude locus on 1q32. J Med Genet 1999; 36: $888-892$.

19 Wong FK, Karsten A, Larson O et al: Clinical and genetic studies of Van der Woude syndrome in Sweden. Acta Odontol Scan 1999; 2: $72-77$.

20 Wong FK, Koillinen H, Rautio J et al: Genetic heterogeneity and exclusion of a modifying locus at 17p11.2-p11.1 in Finnish families with Van der Woude syndrome. J Med Genet 2001; 38: $198-202$.

21 Kruglyak L, Daly MJ, Reeve-Daly MP, Lander ES: Parametric and nonparametric linkage analysis: a unified multipoint approach. Am J Hum Genet 1996; 58: 1347 - 63.
22 Weeks DE, Ott J, Lathrop GM: SLINK: a general simulation program for linkage analysis. Am J Hum Genet 1990; (Suppl) 47: A204.

23 Houdayer C, Soupre V, Rosenberg-Bourgin $\mathrm{M}$ et al: Linkage analysis of 5 novel Van der Woude syndrome kindreds to 1q3241 markers further supports locus homogeneity of the disease trait. Ann Genet 1999; 42: 69-74.

24 Stickle G: (ed): Congenital malformations worldwide. A report from the international clearinghouse for the birth defects monitoring system. Elsevier Science Publishers B.V., Amsterdam, 1991.

25 Vintiner GM, Lo KK, Holder SE, Winter RM, Malcolm S: Exclusion of candidate genes from a role in cleft lip with or without cleft palate: linkage and association studies. J Med Genet 1993; 30: 773-8.

26 Clouthier DE, Williams SC, Yanagisawa H, Wieduwilt M, Richardson JA, Yanagisawa M: Cranial and cardiac neural crest defects in endothelin-A receptor-deficient mice. Development 1998; 125: $813-824$.

27 Muragaki Y, Mariman ECM, van Beersum SEC et al: A mutation in the gene encoding the alpha-2 chain of the fibril-associated collagen IX, COL9A2, causes multiple epiphyseal dysplasia (EDM2). Nature Genet 1996; 12: $103-105$.

28 Fogh-Andersen P: Inheritance of harelip and cleft palate. Copenhagen, Busck, 1942.

29 Christensen K, Mitchell LE: Familial recurrence-pattern analysis of non-syndromic isolated cleft palate-a Danish registry study. Am J Hum Genet 1996; 58: 182-190. 